Ambiente \& Água - An Interdisciplinary Journal of Applied Science
ISSN 1980-993X - doi:10.4136/1980-993X
www.ambi-agua.net
E-mail: ambi.agua@gmail.com

\title{
Carcinofauna como indicador da integridade biótica de um ambiente estuarino no litoral de Santa Catarina, Brasil
}

\author{
doi: 10.4136/ambi-agua.1540
}

Received: 13 Oct. 2014; Accepted: 26 Dec. 2014

\author{
Fabiane Fisch*; Joaquim Olinto Branco; João Thadeu de Menezes \\ Universidade do Vale do Itajaí (UNIVALI), Itajaí, SC, Brasil \\ Programa de Pós-graduação em Ciência e Tecnologia Ambiental \\ *Autor correspondente: e-mail: fabianebarragens@hotmail.com, \\ branco@univali.br, thadeu@univali.br
}

\section{RESUMO}

Inúmeros métodos são utilizados para calcular índices de integridade biótica (IIB) em diferentes regiões do mundo. O objetivo deste trabalho foi verificar a variação da qualidade ambiental de uma região estuarina, no litoral de Santa Catarina, com base no cálculo do IIB que utiliza como parâmetros dados da comunidade de crustáceos que ocorrem na área. Os dados utilizados foram coletados por diversos autores para o período de 2000 até 2005 e comparados com os dados obtidos por este estudo para os anos de 2012 a 2013, utilizando os mesmos métodos de coleta dos anteriores. Para o cálculo do IIB foram escolhidos 14 atributos inerentes a carcinofauna local e definidas quatro categorias de integridade biótica (muito pobre, pobre, regular e bom). Os valores do IIB foram calculados para cada período e classificados em diferentes categorias para reconhecer suas variações temporais. Os valores obtidos oscilaram ao longo do período amostral, sendo maiores nos anos de 2000-2001, resultando em um valor equivalente a categoria regular, alterando para pobre em 2001-2003, regular entre 2003-2004 e pobre entre 2004-2005, regressando a categoria regular em 2012-2013. Os valores do IIB, encontrados para a região do Saco da Fazenda, refletem a situação de instabilidade da área, característica de ambientes estuarinos, quando avaliados em escalas temporais.

Palavras-chave: índice, qualidade ambiental, Sul do Brasil.

\section{Crustaceans as indicators of biotic integrity of a coastal estuarine area of Santa Catarina, Brazil}

\begin{abstract}
Numerous methods are used to calculate indices of biotic integrity (IBI) in different regions of the world. The objective of this study was to determine the variation of the environmental quality of a coastal estuary of Santa Catarina State, based on the IBI calculation, using the data of the area's crustacean community as parameters. The data were collected by several authors for the period 2000 to 2005 and compared with the data obtained in this study for the years 2012 to 2013, using the same methods of the previous collections. To calculate the IBI, 14 attributes inherent to local crustaceans were chosen and four categories of biotic integrity (very poor, poor, regular and good) were defined. The values of
\end{abstract}


IBI were calculated for each period and classified into different categories to distinguish their temporal variations. The values obtained ranged over the sample period, being higher in 2000-2001, resulting in an value equivalent to the regular category, changing to poor in 2001-2003, regular between 2003-2004, poor between 2004-2005 and regressing to the regular category in 2012-2013. The values of IBI found for the "Saco da Fazenda" region reflect the instability of the area, which is characteristic of estuarine environments when evaluated on time scales.

Keywords: environmental quality, index, Southern Brazil.

\section{INTRODUÇÃO}

Estuários são ecossistemas complexos que formam uma interface entre os ambientes de água marinha e continental, sendo utilizados como berçários ou refúgio para peixes, aves, moluscos e crustáceos (Pinto et al., 2009). A proximidade dos estuários com as aglomerações urbanas os tornam locais ideais para o monitoramento das ações antropogênicas (Alve, 1995; Scott et al., 2005). Assim, planos de gestão que incluam indicadores biológicos são vantajosos para o conhecimento do estado de conservação dos ambientes, ao incorporarem condições que facilitam a detecção dos impactos por meio de índices de biointegridade (Bryce et al., 2002; Elliott e Whitfield, 2011), que servem como ferramenta de processos decisórios ao descreverem as pressões que afetam os ecossistemas ou avaliando o estado do ambiente em relação a ações dos gestores (Pinto et al., 2009).

Neste sentido, a carcinofauna pode ser utilizada como um bioindicador por que: fornece índices ecológicos decorrentes de seus ciclos de vida curtos; responde rapidamente a modificações ambientais (Boltovosky, 1999); vive e se alimenta no interior ou sobre os sedimentos e tendem a acumular as toxinas que são incorporadas na cadeia alimentar; é sensível a estressores presentes na água; é fonte primária de alimento para muitos peixes; e contribui na degradação da matéria orgânica e na ciclagem dos nutrientes (Reynoldson, 1987; Schindler 1987; Reice e Wohlenberg, 1993; Reece e Richardson, 2000).

$\mathrm{O}$ uso de índices de diversidade como bioindicadores tem diminuído pela dificuldade no estabelecimento de uma relação causa-efeito entre a estabilidade dos ecossistemas e a sua diversidade (Washington, 1984). Em sua maioria são calculados com base na riqueza ou distribuição da abundância de espécies e não consideram o tipo de organismo presente e a capacidade de se adaptar as mudanças no meio. A utilização de índices de integridade biótica alterou o "conceito" de organismo indicador que foi substituído por comunidade indicadora (Segnini, 2003). A integridade biótica é então compreendida como uma escala de condições biológicas de áreas muito perturbadas em relação às áreas com pouca ou nenhuma intervenção, (Karr, 1999).

Entre as vantagens deste índice estão a facilidade na interpretação dos resultados já que a informação é resumida em um único valor que é comparável a uma referência e assim possibilita uma maior "compreensão" dos tomadores de decisões, diferentes níveis de organização ecológica estão ordenados em uma única medida, a metodologia tem custo baixo o que facilita a sua execução e reflete as respostas biológicas em relação às intervenções antropogênicas. Como desvantagem a subjetividade no estabelecimento das variáveis que definem conceitualmente a integridade biótica, pois dificultam o entendimento entre as modificações naturais e as induzidas pelo homem (Fore et al., 1996; Karr e Chu, 1999).

Mas toda esta informação sobre um determinado ambiente precisava ser organizada e integrada de maneira que fosse possível a identificação dos fatores que a alteravam. Karr (1981) propôs então um cálculo do Índice de Integridade Biótica para a ictiofauna de um riacho e indicou as variáveis que poderiam afetar a sua integridade biótica (composição, riqueza de espécies e fatores ecológicos). Ele estabeleceu seis categorias de integridade 
(excelente, bom, ruim, regular, muito pobre, ausência de peixes) e para cada variável foi atribuído um valor $(1,3,5)$ que somados indicariam uma das categorias de qualidade do ambiente.

Esta primeira proposta foi então modificada e utilizada em ambientes marinhos (Jameson et al., 2001), estuarinos (Breine et al., 2008), lacustres (Karr e Dionne, 1991) e terrestres (O’Connell et al., 2007) e também agregando outros componentes da biota aquática como macroinvertebrados (Couceiro et al., 2012) e anfíbios (Simon et al., 2000).

No Brasil os Índices de Integridade Biótica (IIB) foram propostos para ecossistemas de água doce (Araújo, 1998; Bozzetti e Schulz, 2004; Ferreira e Cassati, 2006; Ferreira e Flynn, 2012), estuários (Soares et al., 2011), como ferramenta para diagnosticar a integridade ambiental costeira, aplicado a Baixada Santista/SP (Bolta e Flynn, 2013) e para fragmentos florestais, utilizando aves como indicadores (Anjos et al., 2009).

Embora a utilização de crustáceos para a caracterização da integridade biótica de um ecossistema não seja recorrente (Gabriels et al., 2010), para o grupo dos braquiúros a variedade de espécies em determinadas regiões pode indicar a existência de ambientes favoráveis ao seu desenvolvimento e o seu estudo servir como base na definição de mecanismos de conservação, monitoramento e manutenção da biodiversidade (Braga et al., 2005).

O objetivo deste trabalho foi verificar a variação da qualidade ambiental de uma região estuarina, no litoral de Santa Catarina, utilizando-se o Índice de Integridade Biótica (Karr, 1981), utilizando como parâmetros para este cálculo dados da comunidade de crustáceos que ocorrem na área, coletados por diversos autores para o período de 2000 até 2005 (Branco e Freitas Jr., 2009; Branco et al., 2011; Freitas Jr. et al., 2013) e comparados com os dados obtidos por este estudo para os anos de 2012 a 2013, utilizando os mesmos métodos de coleta.

\section{MATERIAL E MÉTODOS}

\section{1. Área de estudo}

A área de estudo, localizada no Saco da Fazenda (denominada como baía Afonso Wippel) (Itajaí, 2004), é um corpo de água costeiro, na área urbana do município de Itajaí/SC (Figura 1), com uma área de aproximadamente $0,7 \mathrm{~km}^{2}$, submetido ao regime de micro-marés misto e hegemonia semidiurna, apresenta variação do nível de água entre 0,4 e 1,2 m para períodos de maré de quadratura e sizígia, respectivamente (Schettini, 2008). O Saco da Fazenda foi formado pelas obras de retificação e fixação do canal de acesso ao Porto de Itajaí (Vargas, 1935) e está inserido no sistema estuarino da foz do rio Itajaí-Açu, recebendo aporte de diversos pequenos tributários, tais como o Ribeirão Schneider. O Saco da Fazenda apresenta uma tendência a acumular sedimentos provenientes do rio Itajaí-Açu e do Ribeirão Schneider (Schettini, 2008). A ocupação desordenada de suas margens origina o lançamento direto de grandes quantidades de efluentes devido à inexistência de uma rede de escoamento sanitário (Theis e Fernandes, 2002). O Saco da Fazenda é uma unidade de conservação municipal (Itajaí, 2008), gerida pela Fundação Municipal de Meio Ambiente de Itajaí.

No ano de 2000 foram iniciadas obras de revitalização no local com a dragagem e desassoreamento, que foi concluída no ano de 2003 e acarretou em modificações ambientais ao ecossistema. O volume dragado foi de $627.518,4 \mathrm{~m}^{3}$ com uma redução entre 20 a $25 \%$ na toxicidade do sedimento em relação ao início das obras, porém a qualidade da água permaneceu a mesma (Araújo et al., 2009). 


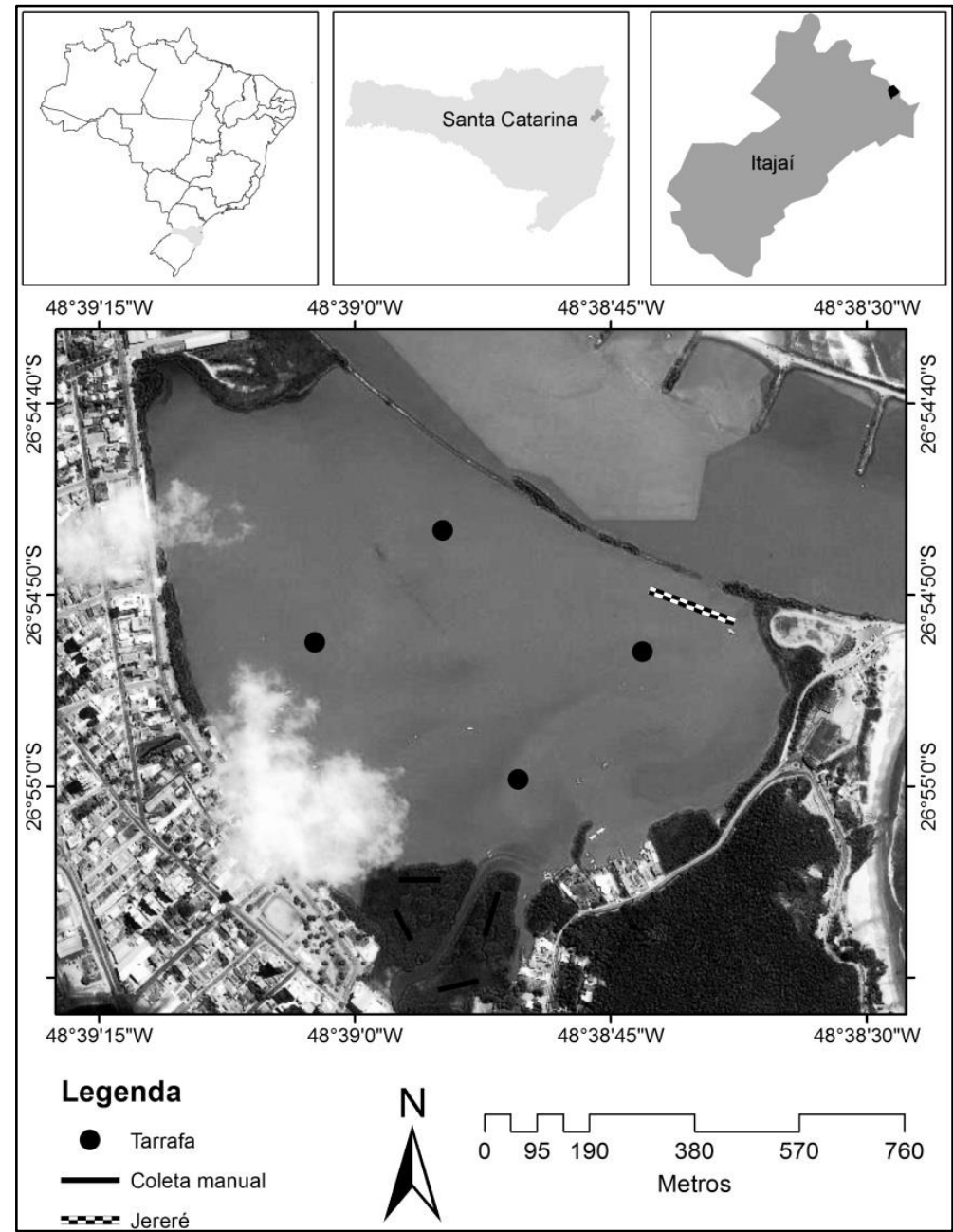

Figura 1. Localização geográfica do Saco da Fazenda e áreas de amostragem com os respectivos métodos - tarrafa, coleta manual e jereré (imagem modificada de Google Earth).

\subsection{Coleta de dados e análises}

Para o cálculo do IIB, para o Saco da Fazenda, foram utilizadas as bases de dados de trabalhos pretéritos da carcinofauna local (Branco e Freitas Jr., 2009; Branco et al., 2011; Freitas Jr. et al., 2013), que abrangeram o período de 2000 até 2005 e com metodologia similares que possibilitam a sua comparação com este estudo.

Além desta base de dados, foram realizadas amostragens trimestrais durante o período de maio de 2012 a abril de 2013. Os locais de coleta foram escolhidos em razão dos elementos naturais da área de forma a se obter uma maior representatividade dos diversos ambientes, bem como repetir os métodos utilizados nos estudos anteriores realizados na região (Figura 1).

As capturas dos camarões ocorreram com auxílio de tarrafa $(25 \mathrm{~mm}$ de malha entre nós opostos), sendo realizados 20 lances em cada ponto. Para os siris foram distribuídos 16 jererés ao longo da abertura do canal de navegação, sendo revisados a intervalos de 30 minutos e mantidos submersos por quatro horas. Os caranguejos foram amostrados em quatro retângulos nas áreas de manguezal e marisma na desembocadura do Ribeirão Schneider. Em cada um utilizamos dois cordões de nylon com $10 \mathrm{~m}$ x $1 \mathrm{~m}$ de comprimento, amarrados em estacas de madeira, estendidos paralelamente, de modo que os exemplares avistados fossem capturados manualmente (Figura 1). 
Todo o material coletado foi acondicionado em sacos plásticos etiquetados e mantidos sob refrigeração. No laboratório de Biologia da Universidade do Vale do Itajaí (UNIVALI) o material foi identificado até o nível de espécie, com o auxílio de guias de identificação (Holthuis, 1980; Melo, 1996) e bases de dados online (Palomares e Pauly, 2014; Worms, 2014). O número de espécies, nos diversos períodos avaliados, foi comparado utilizando-se Análise de Variância Unifatorial (ANOVA).

\subsection{O Índice de Integridade Biótica}

Utilizou-se o IIB desenvolvido por Karr (1981), com adaptação das métricas, metodologias e condições de referência, porém os pressupostos e a estrutura conceitual do IIB propostos por aquele autor foram mantidos.

\subsubsection{Condição de referência}

Para o cálculo do IIB para o Saco da Fazenda utilizou-se a informação pretérita mais antiga (análise quali-quantitativa dos crustáceos existentes no Saco da Fazenda entre os anos de 2000-2001) que serviu como hipótese das características de referência do local (Branco e Freitas Jr., 2009). Este método também foi empregado por Bozzetti e Schulz (2004), Ferreira e Cassati (2006) e Zhuo e Chang (2008) para outros sistemas.

\subsubsection{Atributos do índice}

Com base na proposta inicial do IIB de Karr (1981) e Karr et al. (1986) selecionaram-se os mais consistentes para a avaliação da carcinofauna na área e adaptaram-se os diferentes atributos aplicados em IIB que contemplam definições de funções ecológicas das diferentes partes do estuário (Courrat et al., 2009) e sensibilidade a ações antropogênicas (Breine et al., 2008).

Foi estabelecido um conjunto final de 14 atributos (Tabela 1) que melhor caracterizam a carcinofauna e os padrões de degradação do Saco da Fazenda:

a) Composição da comunidade: dentro de cada família, grupos de espécies podem ser mais ou menos vulneráveis às condições do habitat. A presença de espécies com baixa vulnerabilidade pode indicar um ambiente degradado com o aumento da influência antropocênica (Karr, 1981). A vulnerabilidade foi definida associada à história de vida e parâmetros ecológicos da espécie, expressa em uma escala arbitrária de 1 a 100, sendo 100 a mais vulnerável (Cheung et al., 2005; Palomares e Pauly, 2014);

b) Composição trófica: para que se possa estimar a produção e a dinâmica de consumo dentro da estrutura da cadeia trófica. Quando se tem um declínio na qualidade do local, a tendência é que ocorra um aumento no número de espécies onívoras que sugerem uma degradação nas bases alimentares. Por outro lado a ocorrência de espécies carnívoras de topo pode indicar um ambiente com uma comunidade mais diversificada (Karr, 1981);

c) Uso do habitat: a utilização do ambiente durante alguma fase do ciclo de vida da espécie reflete se as condições necessárias para o seu desenvolvimento estão sendo mantidas;

d) Outros: o número de espécies comerciais presentes na área permite inferir a sua capacidade de suporte. As Instruções Normativas do Ministério do Meio Ambiente (Brasil, 2004; 2005) foram utilizadas para reconhecer as espécies de invertebrados ameaçadas de extinção, sobreexplotadas ou ameaçadas de sobreexplotação.

\subsubsection{Pontuação dos atributos}

Para a determinação dos valores de cada atributo utilizou-se como referência os trabalhos de Schleiger (2000) e Ferreira e Cassati (2006) que foram modificados e adaptados para este sistema. 
A pontuação superior indica $75 \%$ ou mais do valor de referência e a inferior $25 \%$ ou menos do valor de referência. Quando se encontrou $75 \%$ do valor de referência atribuiu-se um peso de 5, se as condições são intermediárias (25-75\%) um peso de 3 e se forem $25 \%$ ou menos da condição de referência um peso de 1 (Tabela 1).

O Índice de Integridade Biótica (IIB) para cada ano foi determinado pelo somatório da pontuação obtida por cada atributo que foi dividido pelo total de atributos e classificado em uma das quatro categorias de integridade biótica (Tabela 2).

Tabela 1. Atributos biológicos e pontuação do IIB (adaptado de Karr, 1981; Roth et al., 1996; Ferreira e Cassati, 2006); Número de famílias e espécies (Branco e Freitas Jr., 2009; Branco et al., 2011; Freitas Jr. et al., 2013); Vulnerabilidade (Cheung et al., 2005; Palomares e Pauly, 2014); Composição trófica, uso e habitat (Palomares e Pauly, 2014; Worms, 2014), IN MMA no 5/2004 (Brasil, 2004) e IN MMA no 52/2005 (Brasil, 2005).

\begin{tabular}{|c|c|c|c|c|}
\hline \multirow{2}{*}{\multicolumn{2}{|c|}{ Atributos }} & \multicolumn{3}{|c|}{ Pontuação } \\
\hline & & 5 & 3 & 1 \\
\hline \multirow{4}{*}{$\begin{array}{l}\text { Composição da } \\
\text { comunidade }\end{array}$} & 1. Número de famílias & $>=7$ & $3>n<7$ & $<=3$ \\
\hline & 2. Número de espécies & $>=11$ & $5>\mathrm{n}<11$ & $<=5$ \\
\hline & 3. Número de espécies com baixa vulnerabilidade $<50$ & $<=5$ & $5>n<11$ & $>=11$ \\
\hline & 4. Número de espécies com alta vulnerabilidade $>=50$ & $>=11$ & $5>\mathrm{n}<11$ & $<=5$ \\
\hline \multirow{6}{*}{$\begin{array}{l}\text { Composição } \\
\text { trófica }\end{array}$} & 5. Número de espécies herbívoras & $<=5$ & $5>n<11$ & $>=11$ \\
\hline & 6. Número de espécies herbívoras - detritívoras & $<=5$ & $5>\mathrm{n}<11$ & $>=11$ \\
\hline & 7. Número de espécies detritívoras & $<=5$ & $5>\mathrm{n}<11$ & $>=11$ \\
\hline & 8. Número de espécies detritívoras - onívoras & $<=5$ & $5>n<11$ & $>=11$ \\
\hline & 9. Número de espécies onívoras & $>=11$ & $5>n<11$ & $<=5$ \\
\hline & 10. Número de espécies carnívoras & $>=11$ & $5>n<11$ & $<=5$ \\
\hline \multirow{2}{*}{ Uso do habitat } & $\begin{array}{l}\text { 11. Número de espécies com ciclo de vida } 100 \% \\
\text { estuarino }\end{array}$ & $>=11$ & $5>\mathrm{n}<11$ & $<=5$ \\
\hline & $\begin{array}{l}\text { 12. Número de espécies com ciclo de vida independe } \\
\text { do estuário }\end{array}$ & $<=5$ & $5>n<11$ & $>=11$ \\
\hline \multirow[b]{2}{*}{ Outros } & 13. Número de espécies com uso comercial & $>=11$ & $5>n<11$ & $<=5$ \\
\hline & $\begin{array}{l}\text { 14. Número de espécies registradas nas IN MMA no } \\
5 / 2004 \text { e IN MMA n }{ }^{\circ} 52 / 2005\end{array}$ & $>=11$ & $5>\mathrm{n}<11$ & $<=5$ \\
\hline
\end{tabular}

O IIB foi estabelecido pela média destes valores e classificado em quatro categorias de qualidade de integridade biótica (Ferreira e Cassati, 2006) (Tabela 2).

Tabela 2. Descrição das categorias de integridade biótica (adaptado de Karr, 1981; Roth et al., 1999; Ferreira e Cassati, 2006).

\begin{tabular}{lcl}
\hline Categoria & Valor Numérico & \multicolumn{1}{c}{ Descrição } \\
\hline Bom & $4,0-5,0$ & $\begin{array}{l}\text { Comparável as melhores situações sem a influência do homem } \\
\text { (atributos biológicos > 75\%) da condição referência. }\end{array}$ \\
Regular & $3,0-3,9$ & $\begin{array}{l}\text { Comparável a estuários de referência, mas com aspectos da } \\
\text { biologia comprometida (atributos biológicos entre 75 e 50\%). } \\
\text { Sinais de deterioração adicionais, distantes da situação } \\
\text { minimamente impactada (entre 50 e 25\% da condição } \\
\text { referência). } \\
\text { Pobre }\end{array}$ \\
Muito Pobre & $2,0-2,9$ & $\begin{array}{l}\text { Poucos crustáceos presentes, com atributos biológicos abaixo de } \\
25 \% \text { da condição referência. }\end{array}$ \\
\hline
\end{tabular}




\section{RESULTADOS E DISCUSSÃO}

De acordo com Branco e Freitas Jr. (2009) e Branco et al. (2011) para os diferentes períodos avaliados foram registradas seis famílias de crustáceos (2000-2001), duas famílias (2001-2002), três famílias (2002-2003), cinco famílias (2003-2004) e três famílias (2004-2005). Neste estudo, para o período de 2012-2013, foram registradas cinco famílias (Portunidae, Ocypodidae, Grapsidae, Penaeidae e Palaemonidae) (Tabela 3).

Das 16 espécies registradas, ao longo dos períodos analisados, seis foram capturadas em todas as amostragens, quatro espécies ocorreram em apenas um dos períodos e as demais espécies oscilaram entre duas, quatro e cinco ocorrências ao longo das amostragens (Tabela 3). O número de espécies, nos diversos períodos avaliados, não apresentou diferença significativa (ANOVA: $\mathrm{F}=0,3777, \mathrm{p}=0,8616$ ).

A carcinofauna do Saco da Fazenda quando comparada a pesquisas desenvolvidas em regiões próximas, como na foz do rio Itajaí-Açu (Leite e Pezzuto, 2012; Boss et al., 2012), no manguezal do rio Camboriú (Rodrigues et al., 1994; Boss et al., 2012) e baía da Babitonga (Branco, 1998; Wunderlich et al., 2008; Boos et al., 2012) indica a ocorrência de algumas espécies em comum, apesar das diferenças hidrológicas e metodológicas (Tabela 3).

Para o ano de referência (2000-2001) a contribuição foi de seis famílias e 13 espécies com um valor do IIB de 3,1. Nos anos de 2001-2002 e 2002-2003 ocorreu uma redução no número de famílias (duas e três respectivamente), com sete espécies para cada ano, porém o IIB oscilou entre 2,9 e 2,7. No ano de 2003-2004 ocorreu um incremento no número de famílias $(n=5)$ e de espécies $(n=10)$ e a pontuação do IIB somou 3,3. Para o ano de 2004-2005 ocorreu um novo declínio no número de famílias $(n=3)$, de espécies $(n=8)$ e da pontuação do IIB $(2,9)$. No ano de 2012-2013 mesmo com o registro de cinco famílias e 12 espécies o IIB pontuou em 3,1 .

Os valores de IIB oscilaram ao longo do período amostral, sendo maiores no início da amostragem (2000-2001), resultando em um valor equivalente a categoria regular, alterando para pobre em 2001-2003, regular entre 2003-2004 e pobre entre 2004-2005, para regressar a categoria regular em 2012-2013 (Figura 2). Para o primeiro (2000-2001) e último ano amostral (2012-2013) é possível constatar a ocorrência de uma maior variedade na composição trófica (onívoros, onívoro-detritívora, herbívoro-detritívora, detritívora, carnívoro) e de um maior número de espécies com ciclo de vida estuarino, com exceção do ano de 2003-2004, o que pode ter contribuído para uma melhor integridade biótica (regular) (Tabela 3). Nos anos em que o resultado do IIB atribuiu a área uma categoria pobre (2001-2002; 2002-2003; 2004-2005) a composição trófica foi caracterizada por espécies onívoras, detritívoras e carnívoras e com um ciclo de vida não estuarino (Tabela 3).

Ambientes estuarinos são instáveis em suas escalas temporais e espaciais podendo se tornar estressantes em decorrência de sua interação com fatores: físicos, químicos, geológicos e biológicos (Araújo et al., 2009; Schettini e Truccolo, 2009) porém algumas espécies que possuem alta resistência a poluição podem ser beneficiadas em relação a outras mais sensíveis (Dauvin et al., 2006). Esta "resistência" pode ser inferida para a área deste estudo porque a categoria de integridade biótica regular (2000-2001; 2003-2004; 2012-2013) foi registrada nos anos em que ocorreu o maior número de espécies com vulnerabilidade zero (Tabela 3). 
Tabela 3. Lista das espécies registradas em trabalhos anteriores (* Branco e Freitas Jr., 2009; Branco et al., 2011; Freitas Jr. et al., 2013) e neste trabalho **. Dieta: O: onívoro, D: detritívora, H: herbívoro, C: carnívoro. Uso comercial: S: sim, N: não. Brasil, 2004 (IN MMA no 5) e Brasil 2005 (IN MMA n $n^{\circ}$ 52): S: consta na IN, N: não consta na IN. Ciclo de vida: N: dependente do estuário, $S$ : independente do estuário. Outros estuários a=Branco, 1998; b=Rodrigues et al., 1994; c=Leite e Pezzuto, 2012; d=Boos et al., 2012; e=Wunderlich et al., 2008.

Família/Espécie

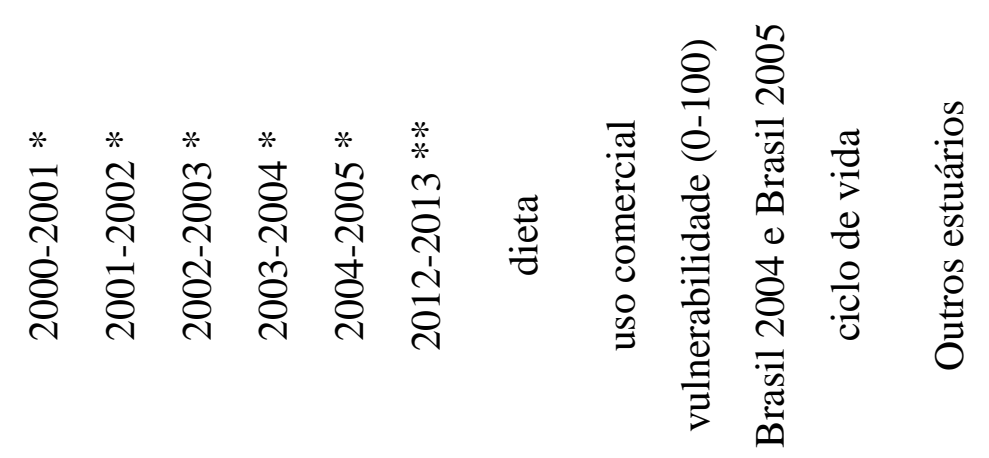

\section{Grapsidae}

Sesarma rectum

Neohelice granulata

\section{Ocypodidae}

Uca uruguayensis

Uca thayeri

Ucides cordatus

Portunidae

Callinectes danae

Callinectes sapidus

Callinectes bocourti

Callinectes ornatus

\section{Penaeidae}

Litopenaeus schmitti

Farfantepenaeus paulensis

Farfantepenaeus brasiliensis

\section{Palaemonidae}

Macrobrachium acanthurus

\section{Alpheidae}

Alpheus spp.

\section{Solenoceridae}

Pleoticus muelleri

Kalliapseudidae

Monokalliapseudes schubarti

\begin{tabular}{lccccccccccccc}
\hline Total de famílias & $\mathbf{6}$ & $\mathbf{2}$ & $\mathbf{3}$ & $\mathbf{5}$ & $\mathbf{3}$ & $\mathbf{5}$ & - & - & - & - & - & - \\
\hline Total de espécies & $\mathbf{1 3}$ & $\mathbf{7}$ & $\mathbf{7}$ & $\mathbf{1 0}$ & $\mathbf{8}$ & $\mathbf{1 2}$ & - & - & - & - & - & - \\
\hline Valor do IIB & $\mathbf{3 , 1}$ & $\mathbf{2 , 9}$ & $\mathbf{2 , 7}$ & $\mathbf{3 , 3}$ & $\mathbf{2 , 9}$ & $\mathbf{3 , 1}$ & - & - & - & - & - & - \\
\hline
\end{tabular}

$\begin{array}{ccccccc}\mathrm{x} & \mathrm{O} & \mathrm{S} & 0 & \mathrm{~N} & \mathrm{~S} & \mathrm{a} \\ \mathrm{x} & \mathrm{O}-\mathrm{D} & \mathrm{S} & 0 & \mathrm{~N} & \mathrm{~S} & - \\ & & & & & & \\ \mathrm{x} & \mathrm{D} & \mathrm{N} & 0 & \mathrm{~N} & \mathrm{~S} & \mathrm{a}, \mathrm{b}, \mathrm{d} \\ \mathrm{x} & \mathrm{D} & \mathrm{N} & 0 & \mathrm{~N} & \mathrm{~S} & \mathrm{a}, \mathrm{b} \\ & \mathrm{H}-\mathrm{D} & \mathrm{S} & 0 & \mathrm{~N} & \mathrm{~S} & \mathrm{a}, \mathrm{b}, \mathrm{d}, \mathrm{e}\end{array}$

\begin{tabular}{|c|c|c|c|c|c|c|c|c|c|c|c|}
\hline X & $\mathrm{X}$ & $\mathrm{X}$ & $\mathrm{x}$ & $X$ & $\mathrm{x}$ & $\mathrm{C}$ & $S$ & 10 & $\mathrm{~N}$ & $\mathrm{~N}$ & $\mathrm{k}$ \\
\hline X & $\mathrm{X}$ & $\mathrm{X}$ & $\mathrm{X}$ & $X$ & $X$ & $\mathrm{O}$ & & 13 & $\mathrm{~S}$ & $\mathrm{~S}$ & \\
\hline & $\mathrm{X}$ & $\mathrm{X}$ & $\mathrm{X}$ & $X$ & $X$ & $\mathrm{C}$ & $S$ & 10 & $\mathrm{~N}$ & $\mathrm{~S}$ & \\
\hline & $\mathrm{x}$ & & $\mathrm{X}$ & $X$ & $\mathrm{X}$ & $\mathrm{C}$ & $S$ & 10 & $\mathrm{~N}$ & S & \\
\hline
\end{tabular}

$\begin{array}{llllllllllll}\mathrm{X} & \mathrm{X} & \mathrm{X} & \mathrm{X} & \mathrm{X} & \mathrm{X} & \mathrm{O} & \mathrm{N} & 10 & \mathrm{~N} & \mathrm{~S} & \mathrm{a}, \mathrm{c}\end{array}$ $\begin{array}{llllllllllll}x & x & x & x & X & x & O & S & 10 & S & N & -\end{array}$

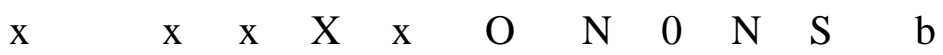
$\begin{array}{llllll}\mathrm{X} & \mathrm{O} & \mathrm{N} & 0 & \mathrm{~N} & \mathrm{~S}\end{array}$ $\begin{array}{llllllllllll}x & x & x & x & X & x & \text { O-D } & S & 10 & S & N & a, b\end{array}$ 


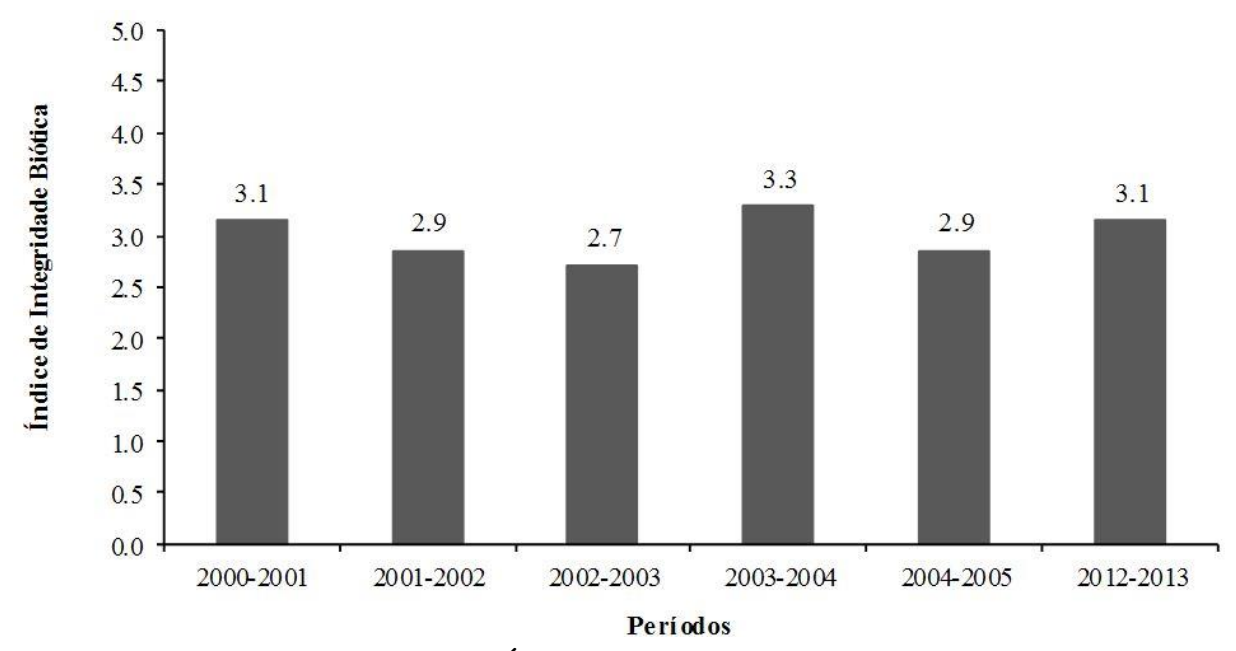

Figura 2. Variação do Índice de Integridade Biótica (IIB) entre os períodos avaliados. O valor acima de cada coluna indica o valor do Índice de IIB para o período.

A carcinofauna do Saco da Fazenda no ano de referência (2000-2001) possivelmente estava adaptada às condições ambientais existentes. Com o início das obras de dragagem o ecossistema alterou a sua hidrodinâmica (Araújo et al., 2009), ocorreu a remoção e re-alocação de sedimentos, a destruição de habitats bentônicos ou asfixia e morte de organismos causados pela ação de sucção do equipamento (Branco e Freitas Jr., 2009) o que pode ter ocasionado uma queda no número de espécies entre os anos de 2001-2003. Com o final da dragagem as condições do ecossistema possivelmente melhoraram o que tornou o ambiente mais favorável ao incremento de novas espécies no ano de 2004-2005. A sinergia de alguns fatores (físicos, químicos, biológicos) pode ter originado um novo declínio no número de espécies registradas no Saco da Fazenda entre 2004-2005. Passados sete anos desta última amostragem, no ano de 2012-2013 as espécies encontradas na área $(n=12)$, com exceção de uma, são as mesmas do ano de referência $(n=13)$ o que permite inferir que houve uma recuperação do ecossistema (Tabela 3 ).

Espécies estuarinas tendem a ser resistentes a processos de dragagem (Whitlatch e Zajac, 1985) com períodos de recuperação que podem ser muito variáveis, com intervalos de semanas ou anos (Jones, 1986). Freitas Jr. et al. (2010), em suas pesquisas na baía Sul em Florianópolis-SC, observaram que as atividades de dragagem não interferiram ou ocasionaram prejuízos a carcinofauna estuarina a longo prazo. Entretanto, Bemvenuti et al. (2005), na região estuarina da lagoa dos Patos-RS, identificaram uma diminuição do número e abundância de espécies como efeito inequívoco da dragagem, ocasionando um impacto na ecologia e economia do ecossitema, limitando sua capacidade de funcionar como uma área de berçário. A queda do número de taxons, abundância e biomassa também foram atribuídas como efeitos diretos de curto prazo das dragagens em outras pesquisas (Sánchez-Moyano e García-Asencio, 2010).

O período analisado, de seis anos de dados, permitiu inferir que as prováveis ações antropogênicas ocorridas no Saco da Fazenda alteraram a integridade local. Segundo Karr (1993) a qualidade da água ou outras condições do ambiente podem resultar na mudança e disponibilidade de muitos recursos alimentares o que direta ou indiretamente pode refletir nos resultados do IIB. Para Eklöv et al. (1998) os valores do IIB podem refletir o progressivo aporte de sedimentos, efluentes domésticos, alteração e ocupação desordenada das margens que geram uma transferência de patologias dentro da cadeia trófica com a eliminação das espécies mais sensíveis. Essas variáveis também são encontradas no Saco da Fazenda de 
forma isolada ou associadas às obras de dragagem podem ter contribuído para os resultados encontrados neste estudo.

\section{CONCLUSÃO}

A partir do cálculo do Índice de Integridade Biótica, para diferentes períodos, foi possível ter uma visão geral da situação do ecossistema do Saco da Fazenda e as variações que está região estuarina teve ao logo dos períodos avaliados. A categoria "regular" $(\mathrm{IIB}=3,1)$ encontrada para a região do Saco da Fazenda, para o período de 2012-2013, reflete adequadamente a situação atual da área de estudo.

Apesar do IIB fornecer bons indicativos do status do ambiente, as decisões de gestores fundamentadas no mesmo devem ser cautelosas, devendo sempre ser utilizados vários outros índices associados e devidamente adaptados a cada região. O próprio IIB não deve ser utilizado indiscriminadamente para qualquer tipo de ambiente, sendo necessário escolher adequadamente os organismos e os parâmetros que irão compor o mesmo, os quais farão parte das métricas a serem utilizadas, que devem ser relevantes para cada estudo, melhorando, desta forma sua sensibilidade e robustez.

Para efeito de comparação, sugere-se que em estudos futuros sejam utilizados outros grupos da fauna, tais como peixes ou avifauna aquática, com seus respectivos parâmetros, para verificar o comportamento do IIB na mesma região com estes organismos.

\section{AGRADECIMENTOS}

F. Fisch agradece à CAPES/PROSUP pela bolsa de doutorado concedida, e J. O. Branco ao Conselho Nacional de Desenvolvimento Científico e Tecnológico/CNPq pela bolsa de produtividade recebida durante a elaboração deste artigo.

\section{REFERÊNCIAS}

ALVE, E. Benthic foraminiferal responses to estuarine pollution: a review. Journal of Foraminiferal Research, v. 25, p. 190-203, 1995.

ANJOS, L.; BOCHIO, G. M.; CAMPOS, J. V.; MCCRATE, G. B.; PALOMINO, F. Sobre o uso de níveis de sensibilidade de aves à fragmentação florestal na avaliação da Integridade Biótica: um estudo de caso no norte do Estado do Paraná, sul do Brasil. Revista Brasileira de Ornitologia, v. 17, n. 1, p. 28-36, 2009.

ARAÚJO, F. G. Adaptação do índice de integridade biótica usando a comunidade de peixes para o Rio Paraíba do Sul. Revista Brasileira de Zoologia, v. 58, p. 547-558, 1998.

ARAÚJO, S. A.; PETERMANN. R. M.; SILVA, F. E.; REIS, F. H.; BRANCO, J. O. 2009. Saco da Fazenda: alterações na paisagem local e processos de dragagem. In: BRANCO, J. O.; LUNARDON-BRANCO, M. J.; BELlOTTO, V. R. (Org.). Estuário do Rio Itajaí-Açú, Santa Catarina: caracterização ambiental e alterações antrópicas. Itajaí: Editora UNIVALI, 2009. p. 63-74.

BEMVENUTI, C. E.; ANGONESI, L. G.; GANDRA, M. S. Effects of dredging operations on soft bottom macrofauna in a harbor in the Patos Lagoon estuarine region of the Southern Brazil. Brazilian Journal Biology, v. 65, p. 573-581, 2005. http://dx.doi.org/10.1590/S1519-69842005000400003 
BOLTA, P. M. P.; FLYNN, M. N. Índice M-AMBI como ferramenta para diagnosticar a integridade ambiental costeira, aplicado a Baixada Santista- SP. RevInter Revista Intertox de Toxicologia, Risco Ambiental e Sociedade, v. 6, n. 1, p. 45-77, 2013.

BOLTOVOSKY, D. South Atlantic zoo-plankton. Leiden: Backhuys Publishers, 1999. v.2 $1706 \mathrm{p}$.

BOOS, H.; BUCKUP, G. B.; BUCKUP, L.; ARAÚJO, P. B.; MAGALHÃES, C.; ALMERÃO; SANTOS, R. A.; MANTELATTO, F. Checklist of the Crustacea from the state of Santa Catarina, Brazil. Check List, v. 8, n. 6, p. 1020-1046, 2012.

BOZZETTI, M.; SCHULZ, U. H. An index of biotic integrity based on fish assemblages for subtropical streams in southern Brazilian. Hydrobiology, v. 529, p. 133-144, 2004. http://dx.doi.org/10.1007/s10750-004-5738-6

BRAGA, A. A.; FRANSOZO, A.; BERTINI, G.; FUMIS, P. B. Composition and abundance of the crabs (Decapoda, Brachyura) off Ubatuba and Caraguatatuba,northern coast of São Paulo, Brasil. Biota Neotropical, v. 5, n. 2, p. 1-34, 2005.

BRANCO, J. O. Carcinofauna. In: Proteção e Controle de Ecossistemas CosteirosManguezal da Baía da Babitonga. Brasília, MMA, 1998. 146 p. (Meio Ambiente; Estudos de Pesca, n. 25)

BRANCO, J. O.; FREITAS JÚNIOR, F. Análise quali-quantitativa dos crustáceos no ecossistema Saco da Fazenda, Itajaí, SC. In: BRANCO, J. O.; LUNARDON-BRANCO, M. J.; BELlOTTO, V. R. (Org.). Estuário do Rio Itajaí-Açu, Santa Catarina: caracterização ambiental e alterações antrópicas. Itajaí: UNIVALI, 2009. p. 180-206.

BRANCO, J. O.; FREITAS JÚNIOR, F.; FRACASSO, H. A. A.; BARBIERI, E. Biodiversidade no estuário do Saco da Fazenda, Itajaí-SC. O Mundo da Saúde, São Paulo, v. 35, n. 1, p. 12-22, 2011.

BRASIL. Ministério do Meio Ambiente. Instrução Normativa n⿳0 5, de 21 de maio de 2004. Dispõe sobre as espécies ameaçadas de extinção e espécies sobreexplotadas ou ameaçadas de sobreexplotação, os invertebrados aquáticos e peixes. Disponível em: <www.ibama.gov.br/.../category/62-_?...4482\%3Ain-mma-n-5-2004>. Acesso em: 25 março 2014.

BRASIL. Ministério do Meio Ambiente. Instrução Normativa no 52, de 8 de novembro de 2005. Altera os Anexos I e II da Instrução Normativa $\mathrm{n}^{\circ} 5$ do Ministério do Meio Ambiente, de 21 de maio de 2004. Disponível em:< www.ibama.gov.br/.../category/62_?...4482\%3Ain-mma-n-5-2004>. Acesso em: 25 março 2014.

BREINE, J.; MAES, J.; STEVENS, M.; SIMOENS, I.; ELLIOTT, M.; HEMINGWAY, K. et al. Habitat needs to realise conservation goals for fish in estuaries: case study of the tidal Schelde. INBO.R. - Research Institute for Nature and Forest, v. 3, p. 1-46, 2008.

BRYCE, S. A.; HUGHES, R. M.; KAUFMANN, P. R. Development of a Bird Integrity Index: Using Bird Assemblages as Indicators of Riparian Condition. Environmental Management, v. 30, n. 2, p. 294-310, 2002.

CHEUNG, W. W. L.; PITCHER, T. J.; PAULY, D. A fuzzy logic expert system to estimate intrinsic extinction vulnerabilities of marine fishes to fishing. Biological Conservation, v. 124, p. 97-111, 2005. http://dx.doi.org/10.1016/j.biocon.2005.01.017 
COUCEIRO, S. R. M.; HAMADA, N.; FORSBERG, B. R.; PIMENTEL, T. P.; LUZ, S. L. B. A macroinvertebrate multimetric index to evaluate the biological condition of streams in the Central Amazon region of Brazil. Ecological Indicators, v. 18, p. 118-125, 2012. http://dx.doi.org/10.1016/j.ecolind.2011.11.001

COURRAT, A.; LOBRY, J.; NICOLAS, D.; LAFFARGUE, P.; AMARA, R.; LEPAGE, M.; GIRARDIN, M.; LE PAPE, O. Anthropogenic disturbance on nursery function of estuarine areas for marine species. Estuarine, Coastal and Shelf Science, v. 81, p. 179190, 2009. http://dx.doi.org/10.1016/j.ecss.2008.10.017

DAUVIN, J. C.; DESROY, N.; JANSON, A. L.; VALLET, C.; DUHAMEL, S. Recent changes in estuarine benthic and suprabenthic communities resulting from the development of harbour infrastructure. Marine Pollution Bulletin, v. 53, p. 80-90, 2006. http://dx.doi.org/10.1016/j.marpolbul.2005.09.020

EKLÖV, A. G.; GREENBERG, L. A.; BRÖNMARK, C. LARSSON, P.; BERGLUD, O. Response of stream fish to improved water quality: a comparison between the $1960 \mathrm{~s}$ and 1990s. Freshwater Biology, v. 40, p. 771-782, 1998. http://dx.doi.org/10.1046/j.1365-2427.1998.00370.x

ELLIOTT, M.; WHITFIELD, A. K. Challenging paradigms in estuarine ecology and management. Estuarine, Coastal and Shelf Science, v. 94, n. 4, p. 306-314, 2011. http://dx.doi.org/10.1016/j.ecss.2011.06.016

FERREIRA, C. P.; CASSATI, L. Stream biotic integrity assessed by fish assemblages in the Upper Rio Paraná basin. Biota Neotropical, v. 6, n. 3, p. 1-25, 2006. http://dx.doi.org/10.1590/S1676-06032006000300002

FERREIRA, G. L.; FLYNN, M. N. Índice biótico BMWP' na avaliação da integridade ambiental do Rio Jaguari-Mirim, no entorno das Pequenas Centrais Hidrelétricas de São Joaquim e São José. RevInter Revista Intertox de Toxicologia, Risco Ambiental e Sociedade, v. 5, n. 1, p. 128-139, 2012.

FREITAS JÚNIOR, F.; CHRISTOFFERSEN, M. L.; BRANCO, J. O. Monitoring of carcinofauna abundance and diversity during eight years of expressway construction in Santa Catarina, Brazil. Latin American Journal of Aquatic Research, v. 38, n. 3, p. 461-473, 2010. http://dx.doi.org/10.3856/vol38-issue3-fulltext-10

FREITAS JÚNIOR, F.; CHRISTOFFERSEN, M. L.; ARAÚJO, J. P.; BRANCO, J. O. Spatiotemporal Distribution and Population Structure of monokalliapseudes schubarti (Tanaidacea: Kalliapseudidae) in an Estuary in Southern Brazil. The Scientific World Journal, v. 2013, p. 1-9, 2013. http://dx.doi.org/10.1155/2013/363187

FORE, L. S.; KARR, J. R.; WISSEMAN, R. W. Assessing invertebrate responses to human activities: evaluating alternative approaches. Journal of the North American Benthological Society, v. 15, n. 2, p.212-231, 1996.

http://links.jstor.org/sici?sici=0887-3593\%28199606\%2915\%3A2\%3C212\%3A

AIRTHA\%3E2.0.CO\%3B2-O

GABRIELS, W.; LOCK, K.; DE PAUW, N.; GOETHALS, P. L. M. Multimetric Macroinvertebrate Index Flanders (MMIF) for biological assessment of rivers and lakes in Flanders (Belgium). Limnologica, v. 40, p. 199-207, 2010.

http://dx.doi.org/10.1016/j.limno.2009.10.001 
HOLTHUIS, L. B. Shrimp and prawns of the world. An annoted catalogue of species of interest to fisheries. FAO Fisheries Synopsis, v. 125, n. 1, p 1-261, 1980.

ITAJAÍ. Decreto Municipal no 4.063 de 2 de abril de 2004. Denomina a baía localizada no bairro Fazenda. Disponível em: <http://portaldocidadao.itajai.sc.gov.br>. Acesso em: 15 julho 2013.

ITAJAÍ. Decreto Municipal $n^{0} 8.513$ de 4 de março de 2008. Dispõe sobre a criação da Unidade de Conservação do Saco da Fazenda. Disponível em: <http://portaldocidadao.itajai.sc.gov.br>. Acesso em: 15 julho 2013.

JAMESON, S. C.; ERDMANN, M. V.; KARR, J. R.; POTTS, K. W. Charting a course toward diagnostic monitoring: a continuing review of coral reef attributes and a research strategy for creating coral reef indexes of biotic integrity. Bulletin of Marine Science, v. 69, p. 701-44, 2001.

JONES, A. R. The effects of dredging and spoil disposal on macrobenthos, Hawkesbury Estuary, N.S.W. Marine Pollution Bulletin, v. 17, n. 1, p. 17-20, 1986. http://dx.doi.org/10.1016/0025-326X(86)90798-8

KARR, J. R. Assessment of biotic integrity using fish communities. Fisheries, v. 6, n. 6, p. 21-27, 1981. http://dx.doi.org/10.1577/1548-8446(1981)006<0021:AOBIUF>2.0.CO;2

KARR, J. R. Measuring biological integrity: lessons from streams. In: WOODLEY, S.; KAY, J.; FRANCIS, G. Ecologycal integrity and management of ecosystems. Otawa: Sanit Lucie Press, 1993. p. 83-103.

KARR, J. R. Defining and measuring river health. Freshwater Biology, v. 41, p. 221-234, 1999. http://dx.doi.org/10.1046/j.1365-2427.1999.00427.x

KARR, J. R., CHU, Y. E. W. Restoring life in running waters: better biological monitoring. Washington, D.C.: Island Press, 1999.

KARR, J. R.; DIONNE, M. Designing surveys to assess biological integrity in lakes and reservoirs. In: UNITED STATES. Environmental Protection Agency Biological criteria: research and regulation. Washington, D.C., 1991. p. 62-72.

KARR, J. R.; FAUSH, K. D.; ANGERMEIER, P. R.; YANT, P. R.; SCHLOSSER, I. J. Assessing biological integrity in running waters: a method and its rationale. Champaign: Illinois Natural History Survey, 1986. 31 p.

LEITE, I. P.; PEZZUTO, P. R. Efeito de um evento extremo de enchente sobre os Decápodos infralitorais do estuário do Itajaí-Açu, SC, BRASIL. Brazilian Journal of Aquatic

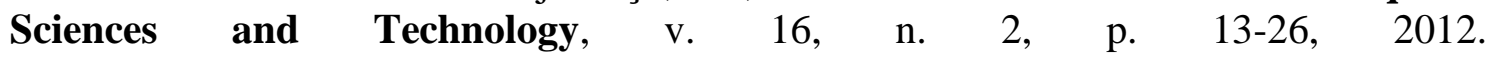
http://dx.doi.org/10.14210/bjast.v16n2.p13-26

MELO, G. A. S. Manual de identificação dos Brachyura (Caranguejos e Siris) do litoral brasileiro. São Paulo: Plêiade, 1996. 604 p.

O'CONNELL, T. J.; BISHOP, A. J.; BROOKS, R. P. Sub-sampling data from the North american breeding bird survey for application to the bird community index, an indicator of ecological condition. Ecological Indicators, v. 7, p. 679-671, 2007. http://dx.doi.org/10.1016/j.ecolind.2006.07.007

PALOMARES, M. L. D.; PAUlY, D. (Eds.). SealifeBase. Disponível em: <www.sealifebase.org>, version (02/2014), Acesso em: 22 janeiro 2014. 
PINTO, R.; PATRÍCIO, J.; BAETA, A.; FATH , B. D.; NETO, J. M.; MARQUES, J. C. Review and evaluation of estuarine biotic indices to assess benthic condition, Ecological Indicators, v. 9, n. 1, p. 1-25, 2009. http://dx.doi.org/10.1016/j.ecolind.2008.01.005

REECE, P.; RICHARDSON, J.S. Biomonitoring with the reference condition approach for the detection of aquatic ecosystem at risk. In: DEARLING, L. M. (Ed.). Proceedings of a conference on the biology and management of species and habitats at risk. Kamloops: University College of the Cariboo, 2000. v. 2. p.549-552.

REICE, S. R.; WOHLENBERG, M. Monitoring freshwater benthic macroinvertebrates and benthic processes: measures for assessment of ecosystem health. In: ROSENBERG, D. M.; RESH, V. H. (Eds.). Freshwater biomonitoring and benthic macroinvertebrates. New York: Chapman and Hall, 1993. p. 287-305.

REYNOLDSON, T. B. Interactions between sediment contaminants and benthic organisms. Hydrobiologia, v. 149, p.53-66, 1987.

RODRIGUES, A. M. T.; PEREIRA, M. T.; WEGNER, P. Z.; BRANCO, J. O.; CLEZAR, L.; HOSTIM-SILVA, M. et al. Manguezal do Rio Camboriú: preservação e controle da qualidade ambiental. Itajaí: IBAMA-CEPSUL, 1994. 65 p.

ROTH, N. E., ALLAN, J. D.; ERICKSON, D. L. Landscape influences on stream biotic integrity assessed at multiple spatial scales. Journal of Landscape Ecology, v. 11, p. 141-156, 1996. http://dx.doi.org/10.1007/BF02447513

SÁNCHEZ-MOYANO, J. E.; GARCÍA-ASENCIO, I. Crustacean assemblages in a polluted estuary from South-Western Spain. Marine Pollution Bulletin, v. 60, p. 1890-1897, 2010. http://dx.doi.org/10.1016/j.marpolbul.2010.07.016

SEGNINI, S. El uso de los macroinvertebrados bentónicos como Indicadores de la condición ecológica de lós cuerpos de agua corriente. Ecotropicos, v. 16, n. 2, p. 45-63, 2003.

SCHETTINI, C. A. F. Hidrologia do Saco da Fazenda, Itajaí, SC. Brazilian Journal of Aquatic Science and Technology, v. 12, n. 1, p. 49-58, 2008. http://dx.doi.org/10.14210/bjast.v12n1.p49-58

SCHETTINI, C. A. F.; TRUCCOLO, E. C. Circulação do baixo estuário do Rio Itajaí, In: BRANCO, J. O.; LUNARDON-BRANCO, M. J.; BELLOTTO, V. R. (Orgs.). Estuário do Rio Itajaí-Açú, Santa Catarina: caracterização ambiental e alterações antrópicas. Itajaí: UNIVALI, 2009. p.13-26.

SCHLEIGER, S. L. Use of an index of biotic integrity to detect effects of land uses on stream fish communities in West-Central Georgia. Transactions of the American Fisheries Society, v. 129, p. 1118-1133, 2000.

http://dx.doi.org/10.1577/1548-8659(2000)129<1118:UOAIOB>2.0.CO;2

SCHINDLER, D. W. Detecting ecosystem responses to anthropogenic stress. Canadian Journal of Fisheries and Aquatic Sciences, v. 44, p. 6-25, 1987.

SCOTT, D. B.; TOBIN, R.; WILLIAMSON, M.; MEDIOLI, F. S.; LATIMER, J. S.; BOOTHMAN,W. A. et al. Pollution monitoring in two North American estuaries: historical reconstructions using benthic foraminifera. Journal of Foraminiferal Research, v. 35, n. 1, p. 65-82, 2005. http://dx.doi.org10.2113/35.1.65 
SIMON, T. P.; JANKOWSKI, R.; MORRIS, C. Modification of an index of biotic integrity for assessing vernal ponds and small palustrine wetlands using fsh, crayfsh, and amphibian assemblages along southern Lake Michigan. Aquatic Ecosystem Health and Management, v. 3, p. 407-418, 2000. http://dx.doi.org/10.1016/S14634988(00)00033-6

SOARES, L. S.; JÚNIOR, M. G. S.; CASTRO, A. C. L.; SAINT-PAUL, U. Comunidade de peixes como Indicador de Qualidade Ambiental de alguns canais de maré do estuário do Rio Paciência, São Luís - MA. Boletim do Laboratório de Hidrobiologia, v. 24, n. 1, p. 01-12, 2011.

THEIS, M. I.; FERNANDES, C. A. Políticas públicas e degradação ambiental em Itajaí, SC. Geosul, v.17, n.33, p 95-116, 2002.

VARGAS, B. M. Relatório da Comissão de Estudos do porto de Itajahy e rio Cachoeira. [S.1. : s.n.], 1935. 135 p.

WASHINGTON, H. G. Diversity, biotic and similarity indices. A review with special relevance to aquatic ecosystems. Water Research, v.18, n. 653-694, 1984.

WHITLATCH, R. B.; ZAJAC, R. N. Biotic interactions among estuarine infaunal opportunistic species. Marine Ecology. Progress Series, v. 21, p. 299-311, 1985.

WORMS. World Register of Marine Species. Disponível em: <http://www.marinespecies.org>. Acesso em: 19 março 2014.

WUNDERLICH, A. C.; PINHEIRO, A. A.; RODRIGUES, A. M. Biologia do caranguejouçá, Ucides cordatus (Crustacea: Decapoda: Brachyura), na Baía da Babitonga, Santa Catarina, Brasil. Revista Brasileira de Zoologia, v. 25, n. 2, p. 188-198, 2008. http://dx.doi.org/10.1590/S0101-81752008000200005

ZHUO, D.; CHANG, J. Annual variations of biotic integrity in the upper Yangtze River using an adapted index of biotic integrity (IBI). Ecological Indicators, v. 8, p. 564-572, 2008. http://dx.doi.org/10.1016/j.ecolind.2007.07.004 